TAMKANG JOURNAL OF MATHEMATICS

Volume 33, Number 3, Autumn 2002

\title{
INTEGRABILITY OF DISTRIBUTIONS IN CR-LIGHTLIKE SUBMANIFOLDS
}

\author{
BAYRAM ȘAHIN AND RIFAT GÜNEȘ
}

\begin{abstract}
In this paper, we study CR-lighlike submanifolds of an indefinite Kaehler manifold. Integrability of distributions on CR-lightlike submanifold investigated. We give some necessary and sufficient conditions on integrability of distibutions on CR-lightlike submanifolds in an indefinite Kaehler manifolds.
\end{abstract}

\section{Introduction}

The study of geometry of CR-submanifolds in a Kaehler manifold was initiated by Bejancu [1] and has been developed by [5], [6] and others. These submanifolds includes the other two classes of submanifolds as special cases.

The study of CR-submanifolds that mentioned above has been confined to differentiable manifolds with positive definite metric. In [8], K. L. Duggal initiated the geometry of CR-submanifolds with a Lorentz metric. The study of CR-submanifolds with a semiRiemann metric continued in [9].

The general theory of a lightlike submanifold (or null)have been developed by Kupeli [7], Bejancu-Duggal, [10], [4]. In [4], the authors constructed the principal vector bundles related to a lightlike submanifold in a semi-Riemann manifold and obtained Gauss-Weingarten formulae as well as other properties of this submanifold.

On the other hand, K. L. Duggal A. Bejancu [12] introduced the notion of a CRlightlike submanifolds in an indefinite Kaehler manifold. Moreover, they have investigated integrability of distributions on real lightlike hypersurfaces of an indefinite Kaehler manifold [11].

The purpose of this paper is to prove and show new results on integrability of distributions on CR-lightlike submanifolds in an indefinite Kaehler manifold.

This paper is organized as follows.

Some preliminary notations and results are given in Section 2. Particular, the basic definition and theorem for lightlike submanifolds and CR-lightlike submanifolds are given.

Received October 31, 2000; revised September 12, 2001.

2000 Mathematics Subject Classification. 53C42.

Key words and phrases. Lightlike Submanifold, CR-Lightlike Submanifold, Indefinite Kaehler Manifold. 
In Section 3, we consider CR-lightlike submannifold of an indefinite Kaehler manifold. Particular, we give some theorems on integrability of distributions on CR-lightlike submanifolds in an indefinite Kaehler manifolds.

\section{Preliminaries}

Let $(\bar{M}, \bar{g})$ be a real $(m+n)$-dimensional semi-Riemann manifold, $m, n>1$ and $\bar{g}$ be a semi-Riemann metric on $\bar{M}$. We denote by $q$ the constant index of $\bar{g}$ and we suppose that $\bar{M}$ is not Riemann manifold.

Let $M$ be a lightlike submanifold of dimension $m$ of $\bar{M}$. In this case there exists a smooth distribution on $M$, named a radical distribution such that $\operatorname{RadT} M_{p}=T M_{p} \cap$ $T M_{p}^{\perp}, \forall p \in M$. If the rank of $\operatorname{RadTM}$ is $r>0, M$ is called an $r$-lightlike submanifold of $\bar{M}$. Then, there are four cases: i); $0<r<\min \{m, n\}$; ii): $1<r=n<m$ iii). $1<r=m<n$ iv). $1<r=m=n$. In the first case the submanifold is called an $r$-lightlike submanifold, in the second a coisotropic submanifold, in thethird an isotropic submanifold and in the fourth a totally lightlike submanifold.

Let $M$ be an r-lightlike submanifold of $\bar{M}$. We consider a complementary distribution $S(T M)$ of $\operatorname{Rad}(T M)$ on $T M$ which is called a screen distribution. Then, we have the direct orthogonal sum

$$
T M=\operatorname{Rad} T M \perp S(T M)
$$

As $S(T M)$ is nondegenerate vector subbundle of $\left.T \bar{M}\right|_{M}$, we put

$$
\left.T \bar{M}\right|_{M}=S(T M) \perp S(T M)^{\perp}
$$

where $S(T M)^{\perp}$ is the complementary orthogonal vector subbundle of $S(T M)$ in $\left.T \bar{M}\right|_{M}$. Moreover $S(T M), S(T M)^{\perp}$ are non-degenerate we have the following orthogonal direct decomposition

$$
S(T M)^{\perp}=S\left(T M^{\perp}\right) \perp S\left(T M^{\perp}\right)^{\perp}
$$

Theorem 2.1.([4]) Let $\left(M, g, S(T M), S\left(T M^{\perp}\right)\right)$ be an r-lightlike submanifold of a semi-Riemannian manifold $(\bar{M}, \bar{g})$. Then, there exists a complementary vector bundle $\operatorname{ltr}(T M)$ called a lightlike transversal bundle of $\operatorname{Rad}(T M)$ in $S\left(T M^{\perp}\right)^{\perp}$ and basis of $\Gamma\left(\left.l \operatorname{tr}(T M)\right|_{U}\right)$ consist of smooth sections $\left\{N_{1}, \ldots, N_{r}\right\}$ of $\left.S\left(T M^{\perp}\right)^{\perp}\right|_{U}$ such that

$$
\bar{g}\left(N_{i}, \xi_{j}\right)=\delta_{i j}, \quad \bar{g}\left(N_{i}, N_{j}\right)=0, \quad i, j=0,1, \ldots, r
$$

where $\left\{\xi_{1}, \ldots, \xi_{r}\right\}$ is a basis of $\left.\Gamma(\operatorname{RadTM})\right|_{U}$.

We consider the vector bundle

$$
\operatorname{tr}(T M)=\operatorname{ltr}(T M) \perp S\left(T M^{\perp}\right) .
$$

Thus

$$
T \bar{M}=T M \oplus \operatorname{tr}(T M)=S(T M) \perp S\left(T M^{\perp}\right) \perp(\operatorname{Rad}(T M) \oplus \operatorname{ltr}(T M)
$$


Now,let $\bar{\nabla}$ be the Levi-Civita connection on $\bar{M}$, we have

$$
\bar{\nabla}_{X} Y=\nabla_{X} Y+h(X, Y), \quad \forall X, Y \in \Gamma(T M)
$$

and

$$
\bar{\nabla}_{X} V=-A_{V} X+\nabla_{X}^{\perp} V, \quad \forall X \in \Gamma(T M)
$$

for any $V \in \Gamma(\operatorname{tr}(T M))$. Using the projectors $L: \operatorname{tr}(T M) \rightarrow l \operatorname{tr}(T M), S: \operatorname{tr}(T M) \rightarrow$ $S\left(T M^{\perp}\right)$. From [12], we have

$$
\bar{\nabla}_{X} Y=\nabla_{X} Y+h^{l}(X, Y)+h^{s}(X, Y)
$$

and

$$
\begin{aligned}
& \bar{\nabla}_{X} N=-A_{N} X+\nabla_{X}^{l} N+D^{s}(X, N) \\
& \bar{\nabla}_{X} W=-A_{W} X+\nabla_{X}^{s} W+D^{l}(X, W)
\end{aligned}
$$

for any $X, Y \in \Gamma(T M), N \in \Gamma(\operatorname{ltr}(T M))$ and $W \in \Gamma\left(S\left(T M^{\perp}\right)\right)$. Where $h^{l}(X, Y)=$ $L h(X, Y), h^{s}(X, Y)=S h(X, Y), \nabla_{X}^{l} N, D^{l}(X, W) \in \Gamma(\operatorname{ltr}(T M)), \nabla_{X}^{s} W, D^{s}(X, N) \in$ $\Gamma\left(S\left(T M^{\perp}\right)\right)$ and $\nabla_{X} Y, A_{N} X, A_{W} X \in \Gamma(T M)$.

Denote by $P$ the projection morphism of $T M$ to the screen distribution, we consider the decomposition

$$
\begin{gathered}
\nabla_{X} P Y=\nabla_{X}^{*} P Y+h^{*}(X, P Y) \\
\triangle_{X} \xi=-A_{\xi}^{*} X+\nabla_{X}^{*^{t}} \xi
\end{gathered}
$$

for any $X, Y \in \Gamma(T M), \xi \in \Gamma(\operatorname{Rad}(T M))$. Then we have the following equations

$$
\begin{gathered}
\bar{g}\left(h^{l}(X, P Y), \xi\right)=g\left(A_{\xi}^{*} X, P Y\right), \quad \bar{g}\left(h^{*}(X, P Y), N\right)=g\left(A_{N} X, P Y\right) \\
g\left(A_{\xi}^{*} P X, P Y\right)=g\left(P X, A_{\xi}^{*} P Y\right), \quad A_{\xi}^{*} \xi=0
\end{gathered}
$$

Let $(\bar{M}, \bar{J}, \bar{g})$ be a real $2 m$-dimensional indefinite almost Hermitian manifold and $M$ be a real $n$-dimensional submanifold of $\bar{M}$.

Definition 2.1.([12]) $A$ submanifold $M$ of an indefinite almost Hermitian manifold $\bar{M}$ is said to be a CR-lightlike submanifold if the following two conditions are fullfilled:

i) $\bar{J}(\operatorname{Rad}(T M))$ is a distribution on $M$ such that

$$
\operatorname{Rad}(T M) \cap \bar{J} \operatorname{Rad}(T M)=\{0\}
$$

ii) There exist vector bundles $S(T M), S\left(T M^{\perp}\right), \operatorname{ltr}(T M), D_{0}$ and $D^{\prime}$ over $M$ such that

$$
S(T M)=\left\{\bar{J}(\operatorname{RadTM}) \oplus D^{\prime}\right\} \perp D_{0}, \quad \bar{J} D_{0}=D_{0}, \quad \bar{J} D^{\prime}=L_{1} \perp L_{2}
$$

where $D_{0}$ is a nondegenarete distribution on $M$ and $L_{1}, L_{2}$ are vector bundles of $l \operatorname{tr}(T M)$ and $S\left(T M^{\perp}\right)$, respectively. 
From by the definition of CR-lightlike submanifold, we have

$$
T M=D \oplus D^{\prime}
$$

where

$$
D=\operatorname{Rad} T M \perp \bar{J} R a d T M \perp D_{0}
$$

We denote by $S$ ve $Q$ the projections on $D$ and $D^{\prime}$, respectively. Then we have

$$
\bar{J} X=f X+\omega X
$$

for any $X, Y \in \Gamma(T M)$. Where $f X=\bar{J} S X$ and $\omega X=\bar{J} Q X$. On the other hand, we set

$$
\bar{J} V=B V+C V
$$

for any $V \in \Gamma(\operatorname{tr}(T M))$. Where $B V \in \Gamma(T M)$ and $C V \in \Gamma(\operatorname{tr}(T M))$.

Unless otherwise stated, $M_{1}$ and $M_{2}$ are supposed to as $\bar{J} L_{1}$ and $\bar{J} L_{2}$, respectively.

\section{Integrability of Distributions}

Definition 3.1. Let $\bar{M}$ be an indefinite Kaehler manifold and $M$ be CR-lightlike submanifold of $\bar{M}$. If $h(X, Y)=0 \forall X, Y \in \Gamma(D)$ then $M$ is called D-geodesic CR-lightlike submanifold.

Theorem 3.1. Let $\bar{M}$ be an indefinite Kaehler manifold and $M$ be CR-lightlike submanifold of $\bar{M}$. If $M$ is D-geodesic then each leaf of holomorphic distribution is total geodesic.

Proof. For $X, Y \in \Gamma(D)$ we have

$$
\begin{aligned}
h(X, Y) & =\bar{\nabla}_{X} Y-\nabla_{X} Y \\
& =-\bar{\nabla}_{X} \bar{J}^{2} Y-\nabla_{X} Y \\
& =-\left(\bar{\nabla}_{X} \bar{J}\right) \bar{J} Y-\bar{J}_{X} \bar{J} Y-\nabla_{X} Y .
\end{aligned}
$$

Since $\bar{M}$ is a Kaehler, we get

$$
h(X, Y)=-\overline{J \nabla}_{X} \bar{J} Y-\nabla_{X} Y .
$$

From (2.7) and (2.14), we derive

$$
\begin{aligned}
h(X, Y) & =-\bar{J} \nabla_{X} \bar{J} Y-\bar{J} h(X, \bar{J} Y)-\nabla_{X} Y \\
& =-f \nabla_{X} \bar{J} Y-\omega \nabla_{X} \bar{J} Y-B h(X, \bar{J} Y)-C h(X, \bar{J} Y)-\nabla_{X} Y .
\end{aligned}
$$

Thus we get

$$
h(X, Y)=-\omega \nabla_{X} \bar{J} Y-C h(X, \bar{J} Y) .
$$


Hence we obtain

$$
\omega \nabla_{X} \bar{J} Y=0
$$

Thus the proof is complete.

From (3.1) we have the following corollary

Corollary 3.2. Let $\bar{M}$ be an indefinite Kaehler manifold and $M$ be CR-lightlike submanifold of $\bar{M}$. Then holomorphic distribution is parallel if and only if $h(X, Y)$ has no component in $\bar{J} D^{\prime}$ for any $X, Y \in \Gamma(D)$.

Lemma 3.3. Let $\bar{M}$ be an indefinite Kaehler manifold and $M$ be CR-lightlike submanifold of $\bar{M}$. Then we have

$$
f[X, Y]=\nabla_{Y} \bar{J} X-\nabla_{X} \bar{J} Y
$$

for any $X, Y \in \Gamma(D)$.

Proof. For any $X, Y \in \Gamma(D)$ we have

$$
h(X, \bar{J} Y)=\bar{\nabla}_{X} \bar{J} Y-\nabla_{X} \bar{J} Y
$$

and

$$
h(\bar{J} X, Y)=\bar{\nabla}_{Y} \bar{J} X-\nabla_{Y} \bar{J} X .
$$

From (3.2) and (3.3) we derive

$$
\begin{aligned}
h(X, \bar{J} Y)-h(\bar{J} X, Y) & =\bar{J}_{X} Y-\bar{J}_{Y} X-\nabla_{X} \bar{J} Y+\nabla_{Y} \bar{J} X \\
& =\bar{J}[X, Y]-\nabla_{X} \bar{J} Y+\nabla_{Y} \bar{J} X \\
& =f[X, Y]+\omega[X, Y]-\nabla_{X} \bar{J} Y+\nabla_{Y} \bar{J} X
\end{aligned}
$$

Taking tangential parts of this equation, we have the proof of lemma.

Theorem 3.4. Let $M$ be a CR-lightlike submanifold of an indefinite Kaehler manifold. Then $D^{\prime}$ is integrable if and only if

$$
A_{\omega W} Z-A_{\omega Z} W \in \Gamma\left(D^{\prime}\right)
$$

for any $Z, W \in \Gamma\left(D^{\prime}\right)$.

Proof. Since $\bar{M}$ is an indefinite Kaehler manifold, we have

$$
\bar{\nabla}_{Z} \bar{J} W=\overline{J \nabla}_{Z} W
$$

for any $Z, W \in \Gamma\left(D^{\prime}\right)$. Thus from (2.7), (2.10) and (2.14) we get

$$
\begin{aligned}
\bar{\nabla}_{Z} f W+\omega W & =\bar{J} \nabla_{Z} W+\bar{J} h(Z, W) \\
-A_{\omega W} Z+\nabla_{Z}^{\perp} \omega W & =f \nabla_{Z} W+\omega \nabla_{Z} W+B h(Z, W)+C h(Z, W) .
\end{aligned}
$$


In similarly we get

$$
-A_{\omega Z} W+\nabla_{W}^{\perp} \omega Z=f \nabla_{W} Z+\omega \nabla_{W} Z+B h(Z, W)+C h(Z, W)
$$

Thus we have

$$
-A_{\omega W} Z+A_{\omega Z} W+\nabla_{Z}^{\perp} \omega W-\nabla_{W}^{\perp} \omega Z=f[Z, W]+\omega[Z, W]
$$

which is proves theorem.

Theorem 3.5. Let $M$ be a CR-lightlike submanifold of an indefinite Kaehler manifold. Then $D_{0}$ is integrable if and only if

i) $\bar{g}\left(h^{*}\left(Z_{0}, Z_{0}^{\prime}\right), N\right)=\bar{g}\left(h^{*}\left(Z_{0}^{\prime}, Z_{0}\right), N\right)$

ii) $\bar{g}\left(h^{*}\left(Z_{0}, \bar{J} Z_{0}^{\prime}\right), N\right)=\bar{g}\left(h^{*}\left(Z_{0}^{\prime}, \bar{J} Z_{0}\right), N\right)$

iii) $h^{s}\left(Z_{0}, \bar{J} Z_{0}^{\prime}\right)=h^{s}\left(\bar{J} Z_{0}, Z_{0}^{\prime}\right)$

iv) $g\left(\nabla_{Z_{0}}^{*} Z_{0}^{\prime}, \bar{J} \xi\right)=g\left(\nabla_{Z_{0}^{\prime}}^{*} Z_{0}, \bar{J} \xi\right)$. for any $Z_{0}, Z_{0}^{\prime} \in \Gamma\left(D_{0}\right), N \in \Gamma(\operatorname{ltr}(T M))$ and $\xi \in$ $\Gamma(\operatorname{Rad}(T M))$.

Proof. We note that to show $D_{0}$ is integrable it is sufficies to show that $\left[Z_{0}, Z_{0}^{\prime}\right] \in$ $\Gamma\left(D_{0}\right)$ for any $Z_{0}, Z_{0}^{\prime} \in \Gamma\left(D_{0}\right)$. By the definition CR-lightlike submanifold $D_{0}$ is integrable if and only if

$$
\bar{g}\left(\left[Z_{0}, Z_{0}^{\prime}\right], N\right)=\bar{g}\left(\left[Z_{0}, Z_{0}^{\prime}\right], \bar{J} N\right)=\bar{g}\left(\left[Z_{0}, Z_{0}^{\prime}\right], \bar{J} W\right)=\bar{g}\left(\left[Z_{0}, Z_{0}^{\prime}\right], \bar{J} \xi\right)=0
$$

for any $Z_{0}, Z_{0}^{\prime} \in \Gamma\left(D_{0}\right), N \in \Gamma(\operatorname{ltr}(T M))$ and $\xi \in \Gamma(\operatorname{Rad}(T M))$. First, From (2.7) and (2.10) we have

$$
\bar{g}\left(\left[Z_{0}, Z_{0}^{\prime}\right], N\right)=\bar{g}\left(h^{*}\left(Z_{0}, Z_{0}^{\prime}\right), N\right)-\bar{g}\left(h^{*}\left(Z_{0}^{\prime}, Z_{0}\right), N\right) .
$$

On the other hand we get

$$
\bar{g}\left(\left[Z_{0}, Z_{0}^{\prime}\right], \bar{J} N\right)=\bar{g}\left(\bar{\nabla}_{Z_{0}} Z_{0}^{\prime}, \bar{J} N\right)-\bar{g}\left(\bar{\nabla}_{Z_{0}^{\prime}} Z_{0}, \bar{J} N\right) .
$$

Using the indefinite Kaehler character of $\bar{M}$, we obtain

$$
\bar{g}\left(\left[Z_{0}, Z_{0}^{\prime}\right], \bar{J} N\right)=-\bar{g}\left(\bar{\nabla}_{Z_{0}} \bar{J} Z_{0}^{\prime}, N\right)+\bar{g}\left(\bar{\nabla}_{Z_{0}^{\prime}} \bar{J} Z_{0}, N\right)
$$

From (2.10) we have

$$
\bar{g}\left(\left[Z_{0}, Z_{0}^{\prime}\right], \bar{J} N\right)=-\bar{g}\left(h^{*}\left(Z_{0}, \bar{J} Z_{0}^{\prime}\right), N\right)+\bar{g}\left(h^{*}\left(Z_{0}^{\prime}, \bar{J} Z_{0}\right), N\right) .
$$

From (2.7) and using the indefinite Kaehler character of $\bar{M}$, we derive

$$
\begin{aligned}
\bar{g}\left(\left[Z_{0}, Z_{0}^{\prime}\right], \bar{J} W\right) & =\bar{g}\left(\bar{\nabla}_{Z_{0}} Z_{0}^{\prime}, \bar{J} W\right)-\bar{g}\left(\bar{\nabla}_{Z_{0}^{\prime}} Z_{0}, \bar{J} W\right) \\
& =-\bar{g}\left(\bar{\nabla}_{Z_{0}} \bar{J} Z_{0}^{\prime}, W\right)+\bar{g}\left(\bar{\nabla}_{Z_{0}^{\prime}} \bar{J} Z_{0}, W\right)
\end{aligned}
$$


and again (2.7) we get

$$
\bar{g}\left(\left[Z_{0}, Z_{0}^{\prime}\right], \bar{J} W\right)=-\bar{g}\left(h^{s}\left(Z_{0}, \bar{J} Z_{0}^{\prime}\right), W\right)+\bar{g}\left(h^{s}\left(\bar{J} Z_{0}, Z_{0}^{\prime}\right), W\right) .
$$

Finally From equation of (2.10) we obatin

$$
\bar{g}\left(\left[Z_{0}, Z_{0}^{\prime}\right], \bar{J} \xi\right)=\bar{g}\left(\nabla_{Z_{0}}^{*} Z_{0}^{\prime}, \bar{J} \xi\right)-\bar{g}\left(\nabla_{Z_{0}^{\prime}}^{*} Z_{0}, \bar{J} \xi\right) .
$$

Thus (3.4),(3.5),(3.6) and (3.7) proof is complete.

Corollary 3.6. Let $M$ be a CR-lightlike submanifold of an indefinite Kaehler manifold. Then $D_{0}$ is integrable if and only if

i) $g\left(Z_{0}^{\prime}, A_{N} Z_{0}\right)=g\left(Z_{0}, A_{N} Z_{0}^{\prime}\right)$

ii) $g\left(\bar{J} Z_{0}^{\prime}, A_{N} Z_{0}\right)=g\left(\bar{J} Z_{0}, A_{N} Z_{0}^{\prime}\right)$

iii) $h^{s}\left(Z_{0}, \bar{J} Z_{0}^{\prime}\right)=h^{s}\left(\bar{J} Z_{0}, Z_{0}^{\prime}\right)$

iv) $\bar{g}\left(h^{l}\left(Z_{0}, \bar{J} Z_{0}^{\prime}\right), \xi\right)=\bar{g}\left(h^{l}\left(Z_{0}, \bar{J} Z_{0}^{\prime}\right), \xi\right)$

for any $Z_{0}, Z_{0}^{\prime} \in \Gamma\left(D_{0}\right), N \in \Gamma(\operatorname{ltr}(T M))$ and $\xi \in \Gamma(\operatorname{Rad}(T M))$

Theorem 3.7. Let $\bar{M}$ be an indefinite Kaehler manifold and $M$ be a CR-lightlike submanifold of $\bar{M}$. Then RadTM is integrable if and only if

i) $\bar{g}\left(h^{l}\left(\xi, \bar{J} \xi^{\prime \prime}\right), \xi^{\prime}\right)=\bar{g}\left(h^{l}\left(\xi^{\prime}, \bar{J} \xi^{\prime \prime}\right), \xi\right)$

ii) $\bar{g}\left(h^{l}\left(\xi, Z_{0}\right), \xi^{\prime}\right)=\bar{g}\left(h^{\prime}\left(\xi^{\prime}, Z_{0}\right), \xi\right)$

iii) $h^{s}\left(\xi^{\prime}, \bar{J} \xi\right)=h^{s}\left(\bar{J} \xi^{\prime}, \xi\right)$

iv) $\bar{g}\left(h^{*}\left(\xi, \bar{J} \xi^{\prime}\right), N\right)=\bar{g}\left(h^{*}\left(\xi^{\prime}, \bar{J} \xi\right), N\right)$.

for any $Z_{0} \in \Gamma\left(D_{0}\right) \xi, \xi^{\prime}, \xi^{\prime \prime} \in \Gamma(\operatorname{Rad}(T M))$ and $N \in \Gamma(l \operatorname{tr}(T M))$. if

Proof. By the definition CR-lightlike submanifold, RadTM is integrable if and only

$$
\bar{g}\left(\left[\xi, \xi^{\prime}\right], \bar{J} \xi^{\prime \prime}\right)=\bar{g}\left(\left[\xi, \xi^{\prime}\right], Z_{0}\right)=\bar{g}\left(\left[\xi, \xi^{\prime}\right], \bar{J} W\right)=\bar{g}\left(\left[\xi, \xi^{\prime}\right], \bar{J} N\right)=0
$$

Thus

$$
\begin{aligned}
\bar{g}\left(\left[\xi, \xi^{\prime}\right], \bar{J} \xi^{\prime \prime}\right) & =\bar{g}\left(\bar{\nabla}_{\xi} \xi^{\prime}, \bar{J} \xi^{\prime \prime}\right)-\bar{g}\left(\bar{\nabla}_{\xi^{\prime}} \xi, \bar{J} \xi^{\prime \prime}\right) \\
& =-\bar{g}\left(\xi^{\prime}, \bar{\nabla}_{\xi} \bar{J} \xi^{\prime \prime}\right)+\bar{g}\left(\xi, \bar{\nabla}_{\xi^{\prime}} \bar{J} \xi^{\prime \prime}\right) \\
& =-\bar{g}\left(h^{l}\left(\xi, \bar{J} \xi^{\prime \prime}\right), \xi^{\prime}\right)+\bar{g}\left(h^{l}\left(\xi^{\prime}, \bar{J} \xi^{\prime \prime}\right), \xi\right)
\end{aligned}
$$

and

$$
\begin{aligned}
\bar{g}\left(\left[\xi, \xi^{\prime}\right], Z_{0}\right) & =\bar{g}\left(\bar{\nabla}_{\xi} \xi^{\prime}, Z_{0}\right)-\bar{g}\left(\bar{\nabla}_{\xi^{\prime}} \xi, Z_{0}\right) \\
& =-\bar{g}\left(A_{\xi^{\prime}}^{*} \xi, Z_{0}\right)+\bar{g}\left(A_{\xi^{*}}^{*} \xi^{\prime}, Z_{0}\right) \\
& =-\bar{g}\left(h^{l}\left(\xi, Z_{0}\right), \xi^{\prime}\right)+\bar{g}\left(h^{l}\left(\xi^{\prime}, Z_{0}\right), \xi\right) .
\end{aligned}
$$


On the other hand we get

$$
\begin{aligned}
\bar{g}\left(\left[\xi, \xi^{\prime}\right], \bar{J} W\right) & =\bar{g}\left(\nabla_{\xi} \xi^{\prime}, \bar{J} W\right)-\bar{g}\left(\bar{\nabla}_{\xi^{\prime}} \xi \bar{J} W\right) \\
& =-\bar{g}\left(W, \bar{\nabla}_{\xi} \bar{J} \xi^{\prime}\right)+\bar{g}\left(W, \bar{\nabla}_{\xi^{\prime}}, \bar{J} \xi\right) \\
& =-\bar{g}\left(h^{s}\left(\xi, \bar{J} \xi^{\prime}\right), W\right)+\bar{g}\left(h^{s}\left(\xi^{\prime}, \bar{J} \xi\right), W\right)
\end{aligned}
$$

and

$$
\begin{aligned}
\bar{g}\left(\left[\xi, \xi^{\prime}\right], \bar{J} N^{\prime \prime}\right) & =\bar{g}\left(\bar{\nabla}_{\xi^{\prime}} \xi, \bar{J} N\right)-\bar{g}\left(\bar{\nabla}_{\xi^{\prime}} \xi\right),(\bar{J} N) \\
& =-\bar{g}\left(N, \bar{\nabla}_{\xi} \bar{J} \xi^{\prime}\right)+\bar{g}\left(W, \bar{\nabla}_{\xi^{\prime}} \bar{J} \xi\right) \\
& =-\bar{g}\left(h^{*}\left(\xi, \bar{J} \xi^{\prime}\right), N\right)+\bar{g}\left(h^{*}\left(\xi^{\prime}, \bar{J} \xi\right), N\right)
\end{aligned}
$$

Thus the proof is complete

Corollary 3.8. Let $\bar{M}$ be an indefinite Kaehler manifold and $M$ be a CR-lightlike submanifold of $\bar{M}$. Then RadTM is integrable if and only if

i) $A_{\xi}^{*} \xi^{\prime} \notin \Gamma\left(M_{1}\right)$

ii) $g\left(\nabla_{\xi^{\prime}}^{*} \bar{J} Z_{0}, \bar{J} \xi\right)=g\left(\nabla_{\xi}^{*} \bar{J} Z_{0}, \bar{J} \xi^{\prime}\right)$

iii) $g\left(A_{W} \xi^{\prime}, \bar{J} \xi\right)=g\left(A_{W} \xi, \bar{J} \xi^{\prime}\right)$

iv) $g\left(A_{N} \xi^{\prime}, \bar{J} \xi\right)=g\left(A_{N} \xi, \bar{J} \xi^{\prime}\right)$

for any $Z_{0} \in \Gamma\left(D_{0}\right) \xi, \xi^{\prime}, \xi^{\prime \prime} \in \Gamma(\operatorname{Rad}(T M))$ and $N \in \Gamma(\operatorname{ltr}(T M))$.

Theorem 3.9. Let $\bar{M}$ be an indefinite Kaehler manifold and $M$ be a CR-lightlike submanifold of $\bar{M}$. Then each leaf of Radical distribution is totally geodesic in $M$ if and only if

i) $A_{\xi}^{*} \xi^{\prime} \notin \Gamma\left(M_{1} \perp D_{0}\right)$

ii) $\bar{g}\left(h^{*}\left(\xi^{\prime}, \bar{J} \xi^{\prime}\right), N\right)=0$

iii) $\bar{g}\left(h^{s}\left(\xi, \bar{J} \xi^{\prime}\right), W\right)=0$

where $M_{1}=\bar{J} L_{1}$.

Proof. By the definition CR-lightlike submanifold, each leaf of Radical distribution is totally geodesic in $M$ if and only if

$$
\bar{g}\left(\nabla_{X} Y, \bar{J} \xi\right)=\bar{g}\left(\nabla_{X} Y, \bar{J} N\right)=\bar{g}\left(\nabla_{X} Y, \bar{J} W\right)=\bar{g}\left(\nabla_{X} Y, Z_{0}\right)=0
$$

$X, Y \in \Gamma(\operatorname{Rad}(T M))$. Thus, from (2.7) and (2.11) we have

$$
\begin{aligned}
\bar{g}\left(\nabla_{X} Y \bar{J} \xi\right) & =\bar{g}\left(\bar{\nabla}_{X} Y, \bar{J} \xi\right)-g(h(X, Y), \bar{J} \xi) \\
& =\bar{g}\left(\bar{\nabla}_{X} Y, \bar{J} \xi\right) \\
& =-\bar{g}\left(Y, \bar{\nabla}_{X} \bar{J} \xi\right) \\
& =\bar{g}\left(\bar{J} Y, \bar{\nabla}_{X} \xi\right) \\
& =-\bar{g}\left(\bar{J} Y, A_{\xi}^{*} X\right)
\end{aligned}
$$


and from (2.7) and (2.10) we get

$$
\begin{aligned}
\bar{g}\left(\nabla_{X} Y, \bar{J} N\right) & =\bar{g}\left(\bar{\nabla}_{X} Y, \bar{J} N\right)-g(h(X, Y), \bar{J} N) \\
& =\bar{g}\left(\bar{\nabla}_{X} Y, \bar{J} N\right) \\
& =\bar{g}\left(\bar{J} Y, \bar{\nabla}_{X} N\right) \\
& =-\bar{g}\left(\bar{J} Y, A_{N} X\right) \\
& =-\bar{g}\left(h^{*}(X, \bar{J} Y), N\right)
\end{aligned}
$$

In similarly we derive

$$
\begin{aligned}
\bar{g}\left(\nabla_{X} Y, \bar{J} W\right) & =\bar{g}\left(\bar{\nabla}_{X} Y, \bar{J} W\right)-g(h(X, Y), \bar{J} W) \\
& =-\bar{g}\left(\bar{\nabla}_{X} \bar{J} Y, W\right)-g(h(X, Y), \bar{J} W) \\
& =\bar{g}\left(\bar{J} Y, \bar{\nabla}_{X} W\right)-g(h(X, Y), \bar{J} W) \\
& =-\bar{g}\left(h^{s}(X, \bar{J} Y), W\right)
\end{aligned}
$$

and

$$
\bar{g}\left(\nabla_{X} Y, Z_{0}\right)=-\bar{g}\left(A_{Y}^{*} X, Z_{0}\right)
$$

from these equations, we have assertion of theorem.

Theorem 3.10. Let $\bar{M}$ be an inndefinite Kaehler manifold and $M$ be a CR-lightlike submanifold of $\bar{M}$. Then $\bar{J} R a d T M$ is integrable if and only if

i) $g\left(\bar{J} \xi, A_{N} \bar{J} \xi^{\prime}\right)=g\left(\bar{J} \xi^{\prime}, A_{N} \bar{J} \xi\right)$

ii) $h^{s}\left(\bar{J} \xi, \xi^{\prime}\right)=h^{s}\left(\bar{J} \xi^{\prime}, \xi\right)$

iii) $g\left(h^{l}\left(\bar{J} \xi, \xi^{\prime}\right), \xi^{\prime \prime}\right)=g\left(h^{l}\left(\xi, \bar{J} \xi^{\prime}\right), \xi^{\prime \prime}\right)$

iv) $g\left(Z_{0}, A_{\xi}^{*} \bar{J} \xi^{\prime}\right)=g\left(Z_{0}, A_{\xi}^{*} \bar{J} \xi\right)$.

Proof. $\bar{J} R a d T M$ is integrable if and only if

$$
\bar{g}\left(\left[\bar{J} \xi^{\prime}, \bar{J} \xi\right], N\right)=\bar{g}\left(\left[\bar{J} \xi^{\prime} \bar{J} \xi\right], \bar{J} W\right)=\bar{g}\left(\left[\bar{J} \xi^{\prime}, \bar{J} \xi\right], \bar{J} \xi^{\prime \prime}\right)=\bar{g}\left(\left[\bar{J} \xi^{\prime}, \bar{J} \xi\right], Z_{0}\right)=0
$$

for any $\left.\xi, \xi^{\prime} \in \Gamma(\operatorname{RadTM}), N \in \Gamma(\operatorname{ltr} T M), W \in \Gamma\left(S(T M)^{\perp}\right)\right)$ and $Z_{0} \in \Gamma\left(D_{0}\right)$. Thus from (2.8) we have

$$
\begin{aligned}
\bar{g}\left(\left[\bar{J} \xi^{\prime}, \bar{J} \xi\right], N\right) & =\bar{g}\left(\bar{\nabla}_{\bar{J} \xi^{\prime}} \bar{J} \xi, N\right)-\bar{g}\left(\bar{\nabla}_{\bar{J} \xi} \bar{J} \xi^{\prime}, N\right) \\
& =-\bar{g}\left(\bar{J} \xi, \bar{\nabla}_{\bar{J} \xi^{\prime}} N\right)+\bar{g}\left(\bar{J} \xi^{\prime}, \bar{\nabla}_{\bar{J} \xi} N\right) \\
& =\bar{g}\left(\bar{J} \xi, A_{N} \bar{J} \xi^{\prime}\right)-\bar{g}\left(\bar{J} \xi^{\prime}, A_{N} \bar{J} \xi\right)
\end{aligned}
$$

and

$$
\begin{aligned}
\bar{g}\left(\left[\bar{J} \xi^{\prime}, \bar{J} \xi\right], \bar{J} W\right) & =\bar{g}\left(\bar{\nabla}_{\bar{J} \xi^{\prime}} \bar{J} \xi, \bar{J} W\right)-\bar{g}\left(\bar{\nabla}_{\bar{J} \xi} \bar{J} \xi^{\prime}, \bar{J} W\right) \\
& =\bar{g}\left(\bar{\nabla}_{\bar{J} \xi^{\prime}} \xi, W\right)-\bar{g}\left(\bar{\nabla}_{\bar{J} \xi} \xi^{\prime}, W\right)
\end{aligned}
$$


or from (2.7) we get

$$
\bar{g}\left(\left[\bar{J} \xi^{\prime}, \bar{J} \xi\right], \bar{J} W\right)=\bar{g}\left(h^{s}\left(\bar{J} \xi^{\prime}, \xi\right), W\right)-\bar{g}\left(h^{s}\left(\bar{J} \xi, \xi^{\prime}\right), W\right)
$$

In similarly we get

$$
\begin{aligned}
\bar{g}\left(\left[\bar{J} \xi^{\prime}, \bar{J} \xi\right], \bar{J} \xi^{\prime \prime}\right) & =\bar{g}\left(\bar{\nabla}_{J \xi^{\prime}} \bar{J} \xi, \bar{J} \xi^{\prime \prime}\right)-\bar{g}\left(\bar{\nabla}_{\bar{J} \xi} \bar{J} \xi^{\prime}, \bar{J} \xi^{\prime \prime}\right) \\
& =\bar{g}\left(\bar{\nabla}_{\bar{J} \xi^{\prime}} \xi, \xi^{\prime \prime}\right)-\bar{g}\left(\bar{\nabla}_{\bar{J} \xi} \xi^{\prime}, \xi^{\prime \prime}\right) \\
& =\bar{g}\left(h^{l}\left(\bar{J} \xi^{\prime}, \xi\right), \xi^{\prime \prime}\right)-\bar{g}\left(h^{l}\left(\bar{J} \xi, \xi^{\prime}\right), \xi^{\prime \prime}\right)
\end{aligned}
$$

and

$$
\begin{aligned}
\bar{g}\left(\left[\bar{J} \xi^{\prime}, \bar{J} \xi\right], Z_{0}\right) & =\bar{g}\left(\bar{\nabla} \bar{J}_{\xi^{\prime}} \bar{J} \xi, Z_{0}\right)-\bar{g}\left(\bar{\nabla} \bar{J}_{\bar{J}} \bar{J} \xi^{\prime}, Z_{0}\right) \\
& =-\bar{g}\left(\bar{\nabla}_{\bar{J} \xi^{\prime}} \xi, \bar{J} Z_{0}\right)+\bar{g}\left(\bar{\nabla} \bar{J}_{\bar{J} \xi} \xi^{\prime}, \bar{J} Z_{0}\right) \\
& =\bar{g}\left(\bar{J} Z_{0}, A_{\xi}^{*} \bar{J} \xi^{\prime}\right)-\bar{g}\left(\bar{J} Z_{0}, A_{\xi^{\prime}} J \xi\right) .
\end{aligned}
$$

from these equations, proof is complete.

Lemma 3.11. Let $\bar{M}$ be an indefinite Kaehler manifold and $M$ be a CR-lightlike submanifold of $\bar{M}$. If $f$ is parallel then

$$
B h(X, Y)=0
$$

for any $Y \in \Gamma(D)$.

Proof. For any $X, Y \in \Gamma(T M)$, form (2.7) and (2.6) we obtain

$$
\left(\bar{\nabla}_{X} f\right) Y=A_{\omega Y} X+B h(X, Y) .
$$

From hypothesis of lemma proof is complete.

Theorem 3.12. Let $\bar{M}$ be an indefinite Kaehler manifold and $M$ be a CR-lightlike submanifold of $\bar{M}$. If $f$ is parallel then each leaf of holomorphic distribution is totally geodesic in $M$.

Proof. Since $f$ is parallel, from lemma we have $B h(X, Y)=0$ for any $X, Y \in \Gamma(D)$. Thus we derive

$$
\begin{aligned}
\bar{J} h(X, Y)-C h(X, Y) & =0 \\
\bar{J}_{X} Y-\bar{J} \nabla_{X} Y-C h(X, Y) & =0 .
\end{aligned}
$$

Since $\bar{M}$ is an indefinite Kaehler manifold we get

$$
\begin{aligned}
\bar{\nabla}_{X} \bar{J} Y-\bar{J} \nabla_{X} Y-C h(X, Y) & =0 \\
\nabla_{X} \bar{J} Y+h(X, \bar{J} Y)-f \nabla_{X} Y-\omega \nabla_{X} Y-C h(X, Y) & =0,
\end{aligned}
$$

Hence we obtain $\nabla_{X} \bar{J} Y=f \nabla_{X} Y$, which is proves our assertion. 
From theorem we have the following corollary.

Corollary 3.13. Let $\bar{M}$ be an indefinite Kaehler manifold and $M$ be a CR-lightlike submanifold of $\bar{M}$. Then holomorphic distribution is parallel if and only if $B h(X, Y)=0$ $X, Y \in \Gamma(D)$.

Theorem 3.14. Let $M$ be a CR-lightlike submanifold of an indefinite Kaehler manifold. Then anti-invariant distribution is parallel if and only if $A_{\bar{J}_{Y}} X$ has no components in holomorphic distribution for any $X, Y \in \Gamma\left(D^{\prime}\right)$.

Proof. From (3.8) we have

$$
-f \nabla_{X} Y=A_{\omega Y} X+B h(X, Y)
$$

for any $X, Y \in \Gamma\left(D^{\prime}\right)$. Hence proof is complete.

Example 3.1. We consider a submanifold $M$ codimension two in $I R_{2}^{8}$ given by the equations

$$
\begin{aligned}
& x_{7}=x_{1} \cos \alpha-x_{2} \sin \alpha-x_{5} x_{6} \tan \alpha \\
& x_{8}=x_{1} \sin \alpha+x_{2} \cos \alpha+x_{5} x_{6},
\end{aligned}
$$

where $\alpha \in I R-\left\{\frac{\pi}{2}+k \pi, k \in Z\right\}$. Then $T M$ is spanned by

$$
\begin{aligned}
& U_{1}=(1,0,0,0,0,0, \cos \alpha, \sin \alpha) \\
& U_{2}=(0,1,0,0,0,0,-\sin \alpha, \cos \alpha) \\
& U_{3}=(0,0,1,0,0,0,0,0) \\
& U_{4}=(0,0,0,1,0,0,0,0) \\
& U_{5}=\left(0,0,0,0,1,0,-x_{6} \tan \alpha, x_{6}\right) \\
& U_{6}=\left(0,0,0,0,0,1,-x_{5} \tan \alpha, x_{5}\right) .
\end{aligned}
$$

This example has been given in [12] for $I R_{2}^{6}$. Then its easy check that submanifold 1-lightlike submanifold of $I R_{2}^{8}$ such that $\operatorname{Rad}(T M)=S p\left\{U_{1}\right\}$. Moreover, using the canonical complex structure of $I R_{2}^{8}$, we see that $U_{2}=J U_{1}$. Thus $\operatorname{JRad}(T M)$ is a distribution on $M$. Hence $M$ is a CR-lightlike submanifold. Then we have vector field of $S\left(T M^{\perp}\right)$ as follows

$$
W=\left(\sin \alpha, \cos \alpha, 0,0,-x_{6},-x_{5}, 0,1\right) .
$$

Now we take a vector field of $S\left(T M^{\perp}\right)^{\perp}$ as follows

$$
\begin{aligned}
V= & \left(t, v, 0,0, \frac{x_{6} \sin \alpha+x_{5} \cos \alpha}{x_{5}^{2}+x_{6}^{2}}, \frac{x_{5} \sin \alpha-x_{6} \cos \alpha}{x_{5}^{2}+X_{6}^{2}},\right. \\
& (1+t) \cos \alpha-v \sin \alpha,(1+t) \sin \alpha+v \cos \alpha),
\end{aligned}
$$


where $t, v$ are constants. Then it is easily check that $\bar{g}\left(V, U_{1}\right)=1$. Thus, by the using

$$
N=\frac{1}{\bar{g}\left(V, U_{1}\right)}\left\{V-\frac{\bar{g}(V, V)}{\bar{g}\left(V, U_{1}\right)} U_{1}\right\}
$$

we have

$$
\begin{aligned}
N= & \left(-\frac{1}{2}\left(\frac{1}{x_{5}^{2}+x_{6}^{2}}+1\right), v, 0,0, \frac{x_{6} \sin \alpha+x_{5} \cos \alpha}{x_{5}^{2}+x_{6}^{2}}, \frac{x_{5} \sin \alpha-x_{6} \cos \alpha}{x_{5}^{2}+x_{6}^{2}},\right. \\
& \left.\frac{1}{2} \cos \alpha-\frac{\cos \alpha}{2\left(x_{5}^{2}+x_{6}^{2}\right)}-v \sin \alpha, \frac{1}{2} \sin \alpha-\frac{\sin \alpha}{2\left(x_{5}^{2}+x_{6}^{2}\right)}+v \cos \alpha\right) .
\end{aligned}
$$

Hence the distribution $D^{\prime}$ is spanned by $J N$ and $J W$. On the other hand we describe that $J U_{3}=U_{4}$. Hence $D_{0}$ is spanned by $\left\{U_{3}, U_{4}\right\}$. Thus we have $D=$ $S p\left\{U_{1}, J U_{1}, U_{3}, U_{4}\right\}$. Now, we can investigate integrability of distributions on M. Firstly, its easily obtained that $D_{0}$ and $D$ are integrable. The bracket of $J N$ and $J W$ is obtained

$$
\begin{aligned}
{[J N, J W]=\frac{\left(x_{6}^{2}-x_{5}^{2}\right)}{\left(x_{5}^{2}+x_{6}^{2}\right)^{2}} \frac{\partial}{\partial x_{2}} } & +\frac{4 x_{5}^{2} x_{6} \cos \alpha+2 x_{6}^{2} x_{5} \sin \alpha-2 x_{5}^{3} \sin \alpha}{\left(x_{5}^{2}+x_{6}^{2}\right)^{2}} \frac{\partial}{\partial x_{5}} \\
& +\frac{-4 x_{6}^{2} x_{5} \cos \alpha+2 x_{5}^{2} x_{6} \sin \alpha-2 x_{6}^{3} \sin \alpha}{\left(x_{5}^{2}+x_{6}^{2}\right)^{2}} \frac{\partial}{\partial x_{6}} \\
& +\frac{\left(x_{5}^{2}-x_{6}^{2}\right) \sin \alpha}{\left(x_{5}^{2}+x_{6}^{2}\right)^{2}} \frac{\partial}{\partial x_{7}}+\frac{\left(x_{6}^{2}-x_{5}^{2}\right) \cos \alpha}{\left(x_{5}^{2}+x_{6}^{2}\right)^{2}} \frac{\partial}{\partial x_{8}}
\end{aligned}
$$

On the other hand, by the definition of $C R$-lightlike submanifold, $D^{\prime}$ is integrable if and only if

$$
\bar{g}([J N, J W], N)=\bar{g}([J N, J W], J N)=\bar{g}\left([J N, J W], U_{4}\right)=\bar{g}\left([J N, J W], U_{3}\right)=0 .
$$

It is easily obtained that $\bar{g}\left([J N, J W], U_{4}\right)=\bar{g}\left([J N, J W], U_{3}\right)=0$. On the other hand we have

$$
\bar{g}([J N, J W], N)=\frac{2 x_{5} x_{6} \cos 2 \alpha+2 x_{5} x_{6}+\left(x_{6}^{2}-x_{5}^{2}\right) \sin 2 \alpha}{\left(x_{5}^{2}+x_{6}^{2}\right)^{2}}
$$

and

$$
\bar{g}([J N, J W], J N)=\frac{-2 x_{5} x_{6} \sin 2 \alpha+\left(x_{6}^{2}-x_{5}^{2}\right) \cos 2 \alpha}{\left(x_{5}^{2}+x_{6}^{2}\right)^{2}}
$$

Thus, from (3.9) and (3.10) we see that $D^{\prime}$ isn't integrable. Otherwise $x_{5}=0$ and $x_{6}=0$. This is impossible.

Remark 3.1. In the case of the real hypersurfaces of an indefinite Kaehler manifold, integrability of the distributions $\operatorname{Rad}(T M), J(\operatorname{Rad}(T M))$ and $D^{\prime}$ are trivial. Because, the dimensions of them are one. However, these distributions may not be integrable in any $C R$-lightlike submanifold. Indeed, we see that the distribution $D^{\prime}$ is not integrable in the Example 3.1. Therefore our results about the integrability of distributions in $C R$ lightlike submanifold more general then the results about the integrability of distributions in real lightlike hypersurfaces of an indefinite Kaehler manifold. 


\section{References}

[1] A. Bejancu, CR-submanifolds of a Kaehler manifold I, Proc. Amer. Math. Soc. 69 (1978), 135-142.

[2] A. Bejancu, CR-submanifolds of a Kaehler manifold II, Trans. Amer. Math. Soc. 250 (1979), 333-345.

[3] A. Bejancu, M. Kon and K. Yano, CR-submanifolds of complex space form, J. D. G. 16 (1981), 137-145.

[4] A. Bejancu and K. L. Duggal, Lightlike submanifolds of semi-riemannian manifolds, Acta Appl. Math. 38 (1995), 197-215.

[5] B. Y. Chen, CR-submanifolds of a Kaehler manifold I, J. D. G 16 (1981), 305-323.

[6] B. Y. Chen, CR-submanifolds of a Kaehler manifold II, J. D. G 16 (1981), 493-509.

[7] D. N. Kupeli, Singular semi-riemannian geometry, Kluwer Dortrecht, (1996).

[8] K. L. Duggal, CR-structures and Lorentzian geometry, Acta. Appl. Math. 7 (1986), 211-223.

[9] K. L. Duggal, Lorentzian geometry of CR-submanifolds, Acta. Appl. Math. 17 (1989), 171-193.

[10] K. L. Duggal A. Bejancu, Lightlike submanifolds of codimension two, Math. J. Toyama Univ. (1992), 59-82.

[11] K. L. Duggal and A. Bejancu, Lightlike CR-hypersurfaces of indefinite Kaehler manifolds, Acta. Appl. Math. 31 (1993), 171-190.

[12] K. L. Duggal and A. Bejancu, Lightlike submanifolds of semi-riemannian manifolds and its applicatoins, Kluwer, Dortrecht, (1996).

Inonu University, Faculty of Sciences and Arts, Department of Mathematics, 4100 Malatya/ Turkey. 